\title{
Preparation and characterization of the Adriamycin-loaded amphiphilic chitosan nanoparticles and their application in the treatment of liver cancer
}

\author{
CHANG-HUA KOU $^{1 *}$, JIN HAN $^{2 *}$, XI-LIN HAN $^{1}$, HUI-JIE ZHUANG $^{1}$ and ZI-MING ZHAO ${ }^{3}$ \\ ${ }^{1}$ Department of Oncological Surgery, Central Hospital of Xuzhou, Xuzhou, Jiangsu 221009; \\ ${ }^{2}$ Jiangsu Key Laboratory of New Drug Research and Clinical Pharmacy; ${ }^{3}$ Department of Pharmacy, \\ Xuzhou Medical College, Xuzhou, Jiangsu 221004, P.R. China
}

Received October 29, 2015; Accepted December 16, 2016

DOI: $10.3892 / \mathrm{ol} .2017 .7210$

\begin{abstract}
In the present study, two nanoparticles including lactose myristoyl carboxymethyl chitosan (LMCC) and algal polysaccharide myristoyl carboxymethyl chitosan (AMCC), were obtained for hepatic-targeted Adriamycin (ADM) drug delivery systems. ADM was successfully loaded into the LMCC or AMCC nanoparticle by dialysis. The release function and liver targeting of the nanoparticles was explored, and it was revealed that ADM release from the nanoparticles was greatest at acidic pH 5.5. ADM-conjugated nanoparticles were readily taken up by HU7 human hepatocellular carcinoma cells, relative to HT22 mouse hippocampal neuron cells in vitro. In vivo, ADM-loaded nanoparticles had significant antitumor efficacy with a $62.7 \%$ inhibition rate, followed by ADM and ADM-AMCC (51.2 and 42.5\%, respectively). The tissue distribution study confirmed that ADM-LMCC had an improved liver delivery efficacy, by comparison with ADM. Furthermore, a series of safety studies, including hemolysis, acute toxicity and organ toxicity, revealed that the ADM-loaded LMCC and AMCC nanoparticles had advantages over the commercially available injectable preparation of Adriamycin hydrochloride, in terms of low toxicity levels and increased tolerated dose. These results indicated that LMCC is a promising carrier for injectable ADM nanoparticle and
\end{abstract}

Correspondence to: Dr Hui-Jie Zhuang, Department of Oncological Surgery, Central Hospital of Xuzhou, 199 Jiefang Nan lu, Xuzhou, Jiangsu 221009, P.R. China

E-mail: zhuanghj163@163.com

Dr Zi-Ming Zhao, Department of Pharmacy, Xuzhou Medical College, 209 Tongshan Road, Xuzhou, Jiangsu 221004, P.R. China

E-mail: xzmc_zm@163.com

"Contributed equally

Key words: antitumor drug delivery system, Adriamycin, hepatoma-target ability, lactose myristoyl carboxymethyl chitosan, algal polysaccharide myristoyl carboxymethyl chitosan
ADM-conjugated nanoparticles may improve the efficacy of ADM by hepatic targeting.

\section{Introduction}

Hepatocellular carcinoma (HCC) is one of the most common types of cancer and the third most common cause of cancer-associated mortality, worldwide $(1,2)$. The highest number of patients with HCC was identified in Asian countries (3). Radical resection surgery is the most effective means of treatment for HCC (4). However, due to the rapid spread of cancer or severe liver dysfunction associated with HCC, $~ 80 \%$ of patients are unable to receive radical resection surgery and the mortality rate is high (5). Previous studies revealed that the five-year survival rate for advanced HCC is only $5 \%(6-8)$; therefore, there is an urgent requirement to research more efficient and safer treatments for HCC. Tumor-targeting drug delivery systems may selectively transport cytotoxic agents to the tumor site by exploiting subtle morphological and physiological differences between healthy and malignant cells (9). Therefore, tumor-targeting drug delivery systems have become one of the most attractive anticancer strategies (10).

Nanopharmaceutics is a branch of nanotechnology that has developed rapidly, mainly involving nanocarrier drugs and nanoscale active ingredient research, which may provide targeted treatment for some diseases (11). Nanocarrier drugs include nano-liposomes, nano-polymeric micelles and nanoparticles, which achieve sustained and targeted release of drugs and effectively improve the water-solubility and safety of drugs (12).

Chitosan is one of the most plentiful biomaterials prepared from N-deacetylation of chitin, and has attracted significant interest in the biotechnology field due to its low toxicity, high biocompatibility and biodegradability (13-15). Additionally, chitosan-based nanoparticles have been exploited as delivery system for liver disease targeting $(16,17)$. However, chitosan has poor solubility in neutral media, leading to low loading capacities for nonionic hydrophobic drugs (18). To overcome these disadvantages, chitosan may be amphiphilically modified by the attachment of both hydrophobic and hydrophilic segments to the backbone (19). Previous studies demonstrated that O-carboxymethyl chitosan (CC) is a water-soluble chitosan 
derivative with favorable biocompatibility, similar to chitosan(20). Lactose has been demonstrated to have a ligand targeting function, including liver disease targeting (21). Alginic acid, a high molecular weight polysaccharide obtained from seaweed, is composed of mannuronic acid and guluronic acid (22); previous studies have demonstrated the presence of mannose receptors in the cell membranes of liver non-parenchymal cells (23). Due to the mannose residues contained in alginic acid and the similarity of its structure to the glycosaminoglycan in the extracellular matrix, low molecular weight algal polysaccharides may have liver targeting properties (24).

In the present study, amphiphilically modified chitosan derivatives, lactose myristoyl carboxymethyl chitosan (LMCC) and algal polysaccharide myristoyl carboxymethyl chitosan (AMCC), were designed and the potential of polymeric micelles composed of LMCC or AMCC as liver-targeting carriers of Adriamycin (ADM) was evaluated. The drug-loading capacity, stability, in vitro drug release and liver targeting were studied in detail. In addition, the safety and cytotoxicity of blank nanoparticles and the ADM-loaded nanoparticles was determined.

\section{Materials and methods}

Materials. Adriamycin hydrochloride $(\mathrm{ADM} \cdot \mathrm{HCl})$ was purchased from Zhejiang Hisun Pharmaceutical Co., Ltd. (Taizhou, China). The HU7 human hepatocarcinoma cell line, H22 murine hepatocarcinoma cell line and the HT22 murine hippocampal neuron cell lines were obtained from the Shanghai Cell Bank of the Chinese Academy of Sciences (Shanghai, China). Dulbecco's modified Eagle's medium (DMEM), fetal bovine serum (FBS), $0.25 \%$ trypsin solution and PBS were provided by Gibco (Thermo Fisher Scientific, Inc., Waltham, MA, USA). Radioimmunoprecipitation lysis assay (RIPA) lysate, BCA protein assay kit, and hematoxylin and eosin (H\&E) staining kit were provided by Beyotime Institute of Biotechnology Co., Ltd. (Hangzhou, China). Rhodamine B isothiocyanate (RBITC) was purchased from Aladdin Reagent Co., Ltd. (Shanghai, China). All other reagents were analytical grade and were used without further purification. Double distilled water was used in the current study.

Kunming (KM) mice ( $\mathrm{n}=90,18-22 \mathrm{~g}, 4$ weeks) were supplied by the Laboratory Animal Centre of Xuzhou Medical University (Xuzhou, China), and raised under specific pathogen-free conditions with a $12 \mathrm{~h}$ light/dark cycle, constant temperature $\left(25^{\circ} \mathrm{C}\right)$ and humidity $(60 \%)$, and free access to standard food and water. New Zealand rabbits $(\mathrm{n}=3,2.5 \mathrm{~kg}$, 12 weeks, female) were supplied by the Laboratory Animal Centre of Xuzhou Medical University (Xuzhou, China), and raised under conventional conditions with a $12 \mathrm{~h}$ light/dark cycle, constant temperature $\left(25^{\circ} \mathrm{C}\right)$ and humidity $(60 \%)$, and free access to standard food and water. All animal experiments were performed according to the Guiding Principles for the Care and Use of Experiment Animals in Xuzhou Medical University.

Preparation and characterization of ADM-loaded nanoparticles

Preparation of ADM. ADM was prepared as previously described (25). Briefly, $10 \mathrm{mg} \mathrm{ADM} \cdot \mathrm{HCl}$ was dissolved in $5 \mathrm{ml}$ dimethylformamide. Appropriate triethylamine (TEA, $5 \mu \mathrm{l}$ ) was added into the solution and the mixture was stirred by a magnetic stirrer overnight in the dark at room temperature. The reaction solution was loaded into a dialysis bag with a molecular weight cut-off (MWCO) of 3,500 Da (Spectrum Laboratories, Inc., Rancho Dominguez, CA, USA) and dialyzed against water ( 3 times, $2 \mathrm{~L}$ per time) for $24 \mathrm{~h}$ in the dark. The suspension in the dialysis bag was filtrated by filter paper and the filter cake was washed three times with water. Subsequent to drying under vacuum at $40^{\circ} \mathrm{C}$ for $24 \mathrm{~h}$, ADM was obtained as a deep red powder.

Preparation of ADM-loaded LMCC and AMCC nanoparticles. LMCC and AMCC were prepared by the Jiangsu Key Laboratory of New Drug Research and Clinical Pharmacy (data not presented). A total of $10 \mathrm{mg}$ ADM and $200 \mathrm{mg}$ LMCC or AMCC were dissolved in dimethyl sulfoxide. The solution was dialyzed (MWCO: $3500 \mathrm{Da}$ ) against water (3 times, 21 per time) for $24 \mathrm{~h}$ in the dark. Following this, the suspension was filtered through a $0.45 \mu \mathrm{m}$ Millipore filter to remove insoluble ADM and ADM-loaded LMCC or ADM-loaded AMCC nanoparticles solution was obtained.

Characterization of ADM-loaded LMCC or AMCC nanoparticles. The morphology of LMCC or AMCC nanoparticles was observed by transmission electron microscopy (TEM) via an H-600A transmission electron microscope (Hitachi Ltd., Tokyo, Japan). The nanoparticle size and zeta potential were further determined using a Nicomp ${ }^{\text {TM }} 380$ ZLS (Particle Sizing Systems, Inc., Port Richey, FL, USA), by measuring the dynamic light scattering in a $1 \mathrm{mg} / \mathrm{ml}$ suspension of ADM-LMCC or ADM-AMCC.

Determination of drug loading and entrapment efficiency. The drug loading capacity (LC) and entrapment efficiency (EE) were determined by quantifying the amount of ADM encapsulated into the LMCC or AMCC nanoparticles using fluorescence analysis. All fluorescence quantitative analyses in this article were performed using a fluorescence spectrophotometer (F4600; Hitachi, Ltd., Tokyo, Japan) at excitation and emission bandwidths of $5 \mathrm{~nm}$. Briefly, $10 \mathrm{mg}$ freeze-dried ADM-LMCC or ADM-AMCC nanoparticles were sonicated in $10 \mathrm{ml} \mathrm{6 \%}$ hydrochloric acid alcohol solution [a mixture of $36 \%$ hydrochloric acid and ethanol (200 proof, anhydrous, $\geq 99.5 \%$; Sigma-Aldrich; Merck KGaA, Darmstadt, Germany) in a volume ratio of 6:94] for $5 \mathrm{~min}$, and centrifuged at 13,400 x g at room temperature for $10 \mathrm{~min}$. The fluorescence of ADM in the supernatant was determined by the fluorescence spectrophotometer (F4600; Hitachi, Ltd.) at excitation and emission wavelengths of 501 and $590 \mathrm{~nm}$, respectively. The LC and EE were calculated according to the standard curve of ADM fluorescent intensity to ADM concentration.

ADM release from nanoparticles in vitro. ADM release from LMCC or AMCC nanoparticles in vitro was detected according to a previously described dialysis method (26). PBS ( $\mathrm{pH} 5.5$ and $7.4,0.15 \mathrm{M})$ containing $0.1 \%(\mathrm{w} / \mathrm{v})$ SDS was used as a release medium, and the release profiles of the nanoparticles were investigated. Briefly, $1 \mathrm{ml}$ of ADM-loaded LMCC or ADM-loaded AMCC nanoparticles were added into a dialysis 
bag (MWCO, 3,500 Da). Subsequently, the end-sealed dialysis bag containing the nanoparticles was placed in $50 \mathrm{ml}$ fresh medium (PBS buffer with $0.1 \%$ SDS) at $37^{\circ} \mathrm{C}$ and $100 \mathrm{r} / \mathrm{min}$ in a shaker (THZ-100; Yiheng Technical Co., Ltd., Shanghai, China). At predetermined intervals, including 0.5, 1, 2, 3, 4, 6 , 8,12 and $24 \mathrm{~h}, 2 \mathrm{ml}$ external release medium was extracted and an equal volume of fresh release medium was added. The amount of released ADM was determined by fluorescence measurement $\left(\lambda_{\mathrm{ex}}=501 \mathrm{~nm}, \lambda_{\mathrm{em}}=590 \mathrm{~nm}\right)$. The release experiments were conducted in triplicate.

\section{Cell uptake of ADM-LMCC nanoparticles}

Preparation of RBIT-labeled LMCC. A total of $0.5 \mathrm{~g} \mathrm{LMCC}$ and $0.5 \mathrm{~g}$ RBITC (Sigma-Aldrich; Merck KGaA) were dissolved in $10 \mathrm{ml} \mathrm{PBS} \mathrm{(pH} \mathrm{7.4).} \mathrm{Following} \mathrm{stirring} \mathrm{for} 24 \mathrm{~h}$ in the dark, the reaction solution was transferred into the dialysis bag (MWCO 3500) and dialyzed against water (6 times, 21 per time) for $48 \mathrm{~h}$ in the dark. The solution in the dialysis bag was freeze dried by a lyophilizer (FD-1-50; Biocool, Ltd., Beijing, China) for $24 \mathrm{~h}$ and RBITC-labeled LMCC (RBITC-LMCC) was obtained as a red powder (27).

Cellular uptake. HU7 cells or HT22 cells were seeded in 24 -well plates at a density of $2.0 \times 10^{5}$ cells/well in $1 \mathrm{ml}$ complete DMEM and cultured at $37^{\circ} \mathrm{C}$ with $5 \% \mathrm{CO}_{2}$ for $24 \mathrm{~h}$. To study the effect of nanoparticles' concentration on uptake, the cells were treated with various concentrations $(150,300$ and $600 \mu \mathrm{g} / \mathrm{ml}$ ) of RBITC-LMCC nanoparticles at $37^{\circ} \mathrm{C}$ and $5 \% \mathrm{CO}_{2}$. At 1, 2 and $4 \mathrm{~h}$, the cells were washed three times with PBS. Fluorescent microimages of the cells were obtained with a fluorescent microscope (Leica DMI4000B; Leica Microsytems GmbH, Wetzlar, Germany). Following observation, the cells were lysed with RIPA lysate following the manufacturer's protocol and centrifuged at $12,000 \mathrm{x} \mathrm{g}$ at $4^{\circ} \mathrm{C}$ for $10 \mathrm{~min}$. The fluorescence intensities of RBITC $\left(\lambda_{\mathrm{ex}}=547 \mathrm{~nm}, \lambda_{\mathrm{em}}=582 \mathrm{~nm}\right)$ in supernatants were determined by the fluorescence spectrophotometer (F4600; Hitachi, Ltd.). Fluorescence intensity was normalized with respect to total protein content. The protein content of the supernatant was determined using BCA protein assay kit according to the method specified by the manufacturer.

Antitumor efficacy in vivo. The in vivo antitumor efficacy of ADM-LMCC and ADM-AMCC was evaluated with a subcutaneous $\mathrm{H} 22$ xenograft tumor model in mouse as previously described (28). KM mice ( $\mathrm{n}=20,18-22 \mathrm{~g}$, female) were injected subcutaneously in the axilla of right anterior limb with $0.2 \mathrm{ml}$ cell suspension, containing $1 \times 10^{6} \mathrm{H} 22$ cells in PBS. After 3 days (day 0), the mice were weighed and randomly divided into four groups $(n=5)$ : (1) negative control group (saline group); (2) positive control group (ADM, $5 \mathrm{mg} / \mathrm{kg}$ ); (3) ADM-LMCC (ADM, $5 \mathrm{mg} / \mathrm{kg}$ ); (4) ADM-AMCC (ADM, $5 \mathrm{mg} / \mathrm{kg}$ ). Drug administrations were performed three times via tail vein injection every other day (day 0,2 and 4). The tumor sizes were measured in all mice at day 0,2, 4 and 6 . The tumor volume was calculated by the following formula: $\left(\mathrm{S}^{2} \times \mathrm{L}\right) / 2$, where $\mathrm{S}$ was the short diameter and $\mathrm{L}$ was the long diameter. At day 8 , all the mice were anesthetized with $5 \%$ chloral hydrate solution ( $380 \mathrm{mg} / \mathrm{kg}$ body weight) by intraperitoneal injection and sacrificed by cervical vertebra dislocation followed by separation and measurement of the tumor block. The antitumor efficacies of each formulation were evaluated by the tumor inhibition rate, which was calculated by the following formula: (1 - tumor weight of the treatment group/tumor weight of the negative control group) $\mathrm{x} 100 \%$. In addition, the separated tumor block was fixed in $4 \%(\mathrm{w} / \mathrm{v})$ paraformaldehyde at $4^{\circ} \mathrm{C}$ for $4 \mathrm{~h}$, washed with PBS and embedded in paraffin. Tissue samples were then cut into $8 \mu \mathrm{m}$ thick sections, which were placed onto gelatin-coated slides and stained with H\&E staining kits following the manufacturer's protocol for histological examination.

Biodistribution. To assess the tissue distribution of ADM-loaded nanoparticles, female KM mice bearing H22 hepatoma $\left(\sim 200 \mathrm{~mm}^{3}\right)$ were weighed and randomly divided into three groups $(\mathrm{n}=12)$ : i) $\mathrm{ADM} \cdot \mathrm{HCl}$ group (ADM, $10 \mathrm{mg} / \mathrm{kg}$ ); ii) ADM-LMCC group (ADM, $10 \mathrm{mg} / \mathrm{kg}$ ); iii) ADM-AMCC group (ADM, $10 \mathrm{mg} / \mathrm{kg}$ ). The ADM $\cdot \mathrm{HCl}$ solution and ADM-loaded nanoparticles were intravenously administrated via tail vein at doses of $10 \mathrm{mg} / \mathrm{kg}$. At $0.5,1$, 4 and $8 \mathrm{~h}$ post-injection ( $\mathrm{n}=3$ at each time point), the mice were anaesthetized and blood was collected by retro-orbital bleeding. Then, the mice were sacrificed and the tumors and major organs (heart, liver, spleen, lungs, and kidneys) were dissected from the mice. Tissue samples were blotted with paper towel, rinsed in saline, blotted to remove excess fluid, weighed and stored at $-20^{\circ} \mathrm{C}$.

ADM plasma concentration was determined by measuring ADM fluorescence. Briefly, $0.2 \mathrm{ml}$ plasma and $0.8 \mathrm{ml}$ of $6 \%$ hydrochloric acid alcohol were mixed by vortexing for $5 \mathrm{~min}$, following which they were separated by centrifugation at $13,400 \mathrm{x} g$ at room temperature for $10 \mathrm{~min}$. Subsequently, $100 \mu \mathrm{l}$ of the supernatant was examined to determine the ADM content using the F4600 fluorescence spectrophotometer at excitation and emission wavelengths of 501 and $590 \mathrm{~nm}$, respectively.

ADM tissue concentration was also determined by the fluorescence method described above. A tissue sample $(0.1 \mathrm{~g})$ was homogenized with $1 \mathrm{ml}$ of $6 \%$ hydrochloric acid alcohol. The homogenate was mixed by vortexing for $5 \mathrm{~min}$. Following centrifugation at $13,400 \mathrm{x}$ g for $10 \mathrm{~min}$ at room temperature, the fluorescent intensity of ADM in the supernatant was determined by the fluorescence spectrophotometer $\left(\lambda_{\mathrm{ex}}=501 \mathrm{~nm}\right.$, $\left.\lambda_{\mathrm{em}}=590 \mathrm{~nm}\right)$. The concentration of ADM in samples were calculated according to the standard curve of ADM fluorescent intensity to ADM concentration (29).

\section{Safety of nanoparticles and ADM-loaded nanoparticles}

Hemolysis test. Rabbit blood was used to test the hemolysis effect of nanoparticles. Blood $(10 \mathrm{ml})$ was obtained via the ear vein from the arteria cruralis of healthy New Zealand rabbits ( $2.5 \mathrm{~kg}, 12$ weeks, female). The red blood cells (RBC) were collected by centrifugation at $402 \mathrm{x} g$ at room temperature for $10 \mathrm{~min}$. The RBC pellets at the bottom of the centrifugation tube were washed 3 times with 0.15 M PBS (pH 7.4). Following repeated washing and centrifugation, an adequate quantity of $0.15 \mathrm{M}$ PBS ( $\mathrm{pH}$ 7.4) was added into the $\mathrm{RBC}$ pellets to give a $2.5 \% \mathrm{RBC}$ suspension, this was then stored at $4^{\circ} \mathrm{C}$. Subsequently, $2.5 \% \mathrm{RBC}$ suspensions were incubated with the equal volume of nanoparticles solutions with different concentrations $(2,4,6,8$ and $10 \mathrm{mg} / \mathrm{ml}$ ) for $1 \mathrm{~h}$ in a $37^{\circ} \mathrm{C}$ water bath. The suspension was 
then centrifuged at $1,610 \mathrm{xg}$ at room temperature for $10 \mathrm{~min}$ to remove intact RBC. The supernatant was collected and analyzed for the presence of released hemoglobin using a spectrophotometer (UV-2450; Shimadzu, Kyoto, Japan) at $541 \mathrm{~nm}$ $(n=3)$. To obtain 0 and $100 \%$ hemolysis, saline and distilled water were added in equal volumes to the RBC suspension, respectively. The degree of hemolysis was calculated with the following equation: Hemolysis ration $(\mathrm{HR} \%)=$ [optical density $\left.\left.(\mathrm{OD})_{\text {sample }}-\mathrm{OD}_{\text {negative control }}\right)\right] \times 100 \% /\left(\mathrm{OD}_{\text {positive control }}-\mathrm{OD}_{\text {negative }}\right.$ control). The turbidity of the samples was compensated for by using a sample solution without RBC as a blank control.

Acute toxicity. To determine the acute toxicity of the nanoparticles, the toxic effects of ADM-LMCC and ADM-AMCC on major organs was examined. The nanoparticle suspension $(100 \mathrm{mg} / \mathrm{ml})$ was obtained by re-dissolving the freeze-dried powder of ADM-LMCC and ADM-AMCC in saline. KM mice (male, $\mathrm{n}=10$; female, $\mathrm{n}=10 ; 18-22 \mathrm{~g}$ ) were housed under normal conditions with free access to food and water. Mice were randomly divided into two groups $(n=10)$, and $0.5 \mathrm{ml}$ ADM-LMCC or ADM-AMCC nanoparticles suspension were intravenously administrated via the tail vein. Mice were observed for two weeks in all groups, and the number of mice that survived was recorded. At day 14, all the mice were sacrificed by cervical vertebra dislocation followed by separation and observation of major organs such as the heart, liver, spleen, lung, kidney and brain.

Statistical analysis. Data are expressed as the mean \pm standard deviation. A one-way analysis of variance followed by Dunnett's post-test was used for statistical analysis. Data analysis was performed using a SPSS 19.0 software package (IBM SPSS, Armonk, NY, USA). P $<0.05$ was considered to indicate a statistically significant difference.

\section{Results}

Synthesis and characterization of ADM-loaded LMCC and $A M C C$. In the present study, ADM was obtained from $\mathrm{ADM} \cdot \mathrm{HCl}$. ADM was incorporated into LMCC and AMCC using the dialysis method. Fig. 1 presents the morphology of the prepared nanoparticles as determined by TEM. The results revealed that the morphology of the prepared nanoparticles was spherical, with a lightly rough surface. The mean size of the LMCC particles was $\sim 20 \mathrm{~nm}$, which was slightly smaller than that of AMCC at $\sim 30 \mathrm{~nm}$.

The size distribution and zeta potential of the nanoparticles with or without ADM were investigated by dynamic light scattering. As presented in Table I, the LMCC and AMCC nanoparticles had a mean particle size of $\sim 67.5$ and $\sim 80.4 \mathrm{~nm}$ respectively, which were generally larger than that obtained by TEM. Following drug loading, the mean particle size of ADM-loaded LMCC and AMCC nanoparticles recorded increased to $\sim 86.8$ and $\sim 102.5 \mathrm{~nm}$, respectively. It was revealed that the LMCC and AMCC nanoparticles have a negative surface charge, with a zeta potential of $\sim-17.5$ and $\sim-18.7 \mathrm{mV}$ (Table I) due to the carboxyl $(-\mathrm{COOH})$ dissociation on the CC. Following drug loading, the zeta potential of the LMCC and AMCC nanoparticles exhibited no significant changes $(\mathrm{P}=0.570$ and 0.679 , respectively).
Table I. Size distribution and zeta potential of the nanoparticles $(n=3)$.

\begin{tabular}{lcc}
\hline Nanoparticles & $\begin{array}{c}\text { Mean diameter } \\
(\mathrm{nm})\end{array}$ & $\begin{array}{c}\text { Zeta potential } \\
(\mathrm{mV})\end{array}$ \\
\hline LMCC & $67.5 \pm 11.1$ & $-17.5 \pm 3.7$ \\
AMCC & $80.4 \pm 13.1$ & $-18.7 \pm 5.4$ \\
ADM loaded LMCC & $86.8 \pm 10.3$ & $-15.8 \pm 2.6$ \\
ADM loaded AMCC & $102.5 \pm 18.9$ & $-17.2 \pm 3.8$ \\
\hline
\end{tabular}

LMCC, lactose myristoyl carboxymethyl chitosan; AMCC, algal polysaccharide myristoyl carboxymethyl chitosan; ADM, Adriamycin.

Table II. Drug-loading and entrapment efficiency of LMCC and $\operatorname{AMCC}(n=3)$.

\begin{tabular}{lcc}
\hline Sample & LC (wt \%) & EE (wt \%) \\
\hline LMCC & $3.8 \pm 0.3$ & $81.6 \pm 1.2$ \\
AMCC & $3.4 \pm 0.2$ & $70.3 \pm 0.6$ \\
\hline
\end{tabular}

LMCC, lactose myristoyl carboxymethyl chitosan; AMCC, algal polysaccharide myristoyl carboxymethyl chitosan; wt, weight; LC, loading capacity; EE, encapsulation efficiency.

Drug-loading and entrapment efficiency of prepared nanoparticles. The drug-loading capacities of specific nanoparticles are listed in Table II. The LC of LMCC and AMCC was $\sim 3.8 \%$ for each. In addition, the EE of ADM for LMCC and AMCC was $\sim 80$ and $\sim 70 \%$, respectively. The results suggest that ADM may be effectively loaded into LMCC or AMCC nanoparticles using the dialysis method.

Results of ADM release from nanoparticles in vitro. The drug release behaviors of nanomedicines from LMCC or AMCC nanoparticles were investigated in PBS at $\mathrm{pH} 5.5$ and 7.4, mimicking the physiological pH levels present in the tumor extracellular microenvironment and normal tissues, respectively. The ADM release rate increased as the $\mathrm{pH}$ decreased from $\mathrm{pH}$ 7.4-5.5 in LMCC and AMCC nanoparticles (Fig. 2). Additionally, ADM-loaded AMCC nanoparticles had lower drug release rates compared with ADM-loaded LMCC nanoparticles, which may be associated with the particle size.

Cell uptake of ADM-LMCC nanoparticles. To evaluate the liver targeting of the prepared nanoparticles, the cellular uptake of LMCC in HU7 and HT22 cells was determined by fluorescent microscopy. LMCC was marked with red fluorescence by RBITC. Fig. 3A demonstrated the fluorescent micro-images of HU7 and HT22 cells co-incubated with RBITC-LMCC nanoparticles or free RBITC solution for 1, 2 and $4 \mathrm{~h}$. As predicted, the fluorescence intensity determined in HU7 cells co-incubated with RBITC-LMCC nanoparticles was significantly higher, compared with the fluorescence in HT22 cells at 2 and $4 \mathrm{~h}(\mathrm{P}<0.01)$, which indicated 
A

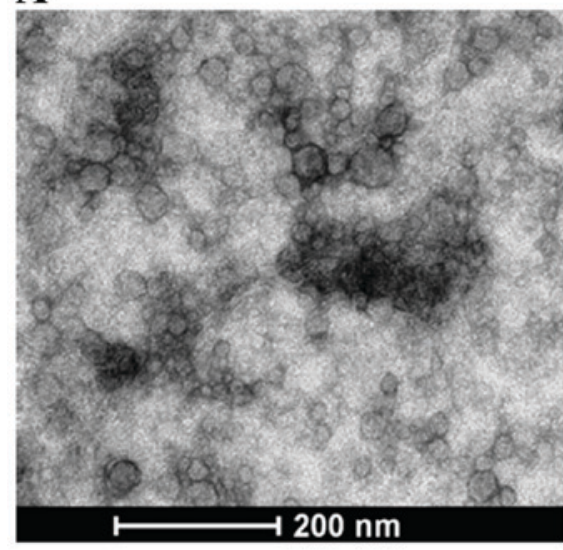

B

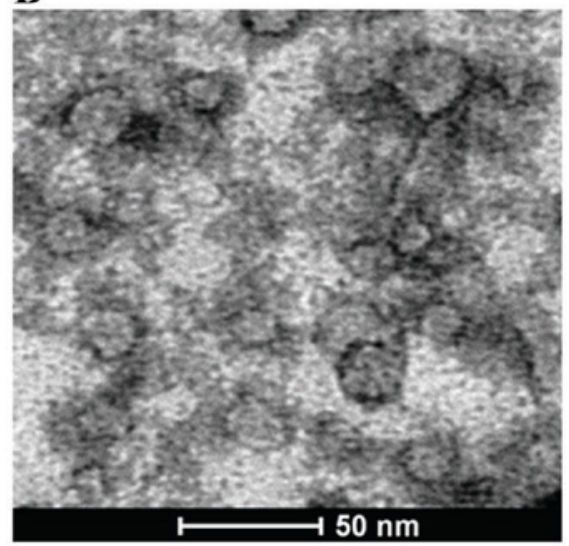

Figure 1. TEM images of (A) LMCC and (B) AMCC. TEM, transmission electron microscopic; LMCC, lactose myristoyl carboxymethyl chitosan; AMCC, algal polysaccharide myristoyl carboxymethyl chitosan.
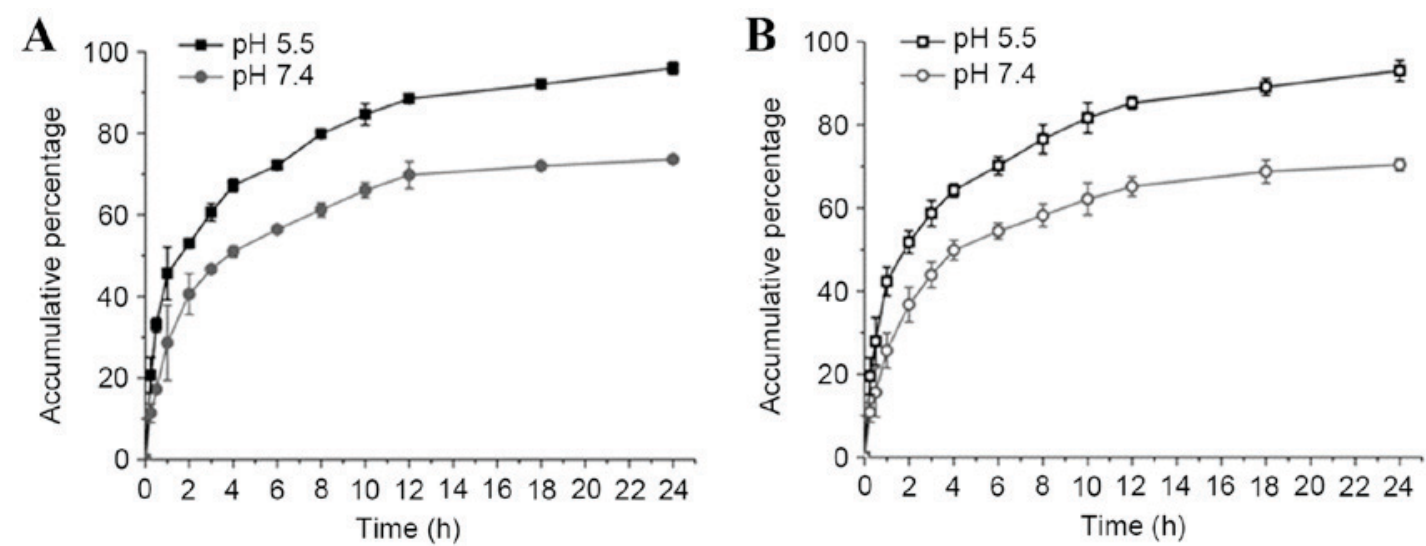

Figure 2. Drug release profiles demonstrating the $\mathrm{pH}$ dependent release of ADM from (A) LMCC and (B) AMCC. ADM, Adriamycin; LMCC, lactose myristoyl carboxymethyl chitosan; AMCC, algal polysaccharide myristoyl carboxymethyl chitosan.

that the prepared nanoparticles had a specific interaction with HU7 cells. Fig. 3B indicated that enhancing the concentration of nanoparticles resulted in significantly increased uptake of RBITC-LMCC in HU7 cells from 150 to $300 \mu \mathrm{g} / \mathrm{ml}(\mathrm{P}<0.01)$. However, the fluorescence intensity did not exhibit a marked improvement when the concentration of nanoparticles was additionally increased to $600 \mu \mathrm{g} / \mathrm{ml}(\mathrm{P}>0.50)$, which resulted in the uptake of RBITC-LMCC by HU7 cells being saturated $>300 \mu \mathrm{g} / \mathrm{ml}$. In addition, the highest intensity of the fluorescence in HU7 cells occurring at $4 \mathrm{~h}$, indicating that the uptake of LMCC nanoparticles was time-dependent.

Antitumor efficacy in vivo. The evaluations of the hepatoma-targeting ability and antitumor efficacy of ADM-loaded nanoparticles in vivo were performed using an $\mathrm{H} 22$ xenograft mouse model. During the complete experimental period (6 days), the weight of the mice body was determined and no significant body weight loss was observed following the administration of ADM or ADM-loaded nanoparticles compared with the initial body weights of the tumor-bearing mice, indicating that the nanoparticles were well tolerated at the dosage level used.

The tumor volume was monitored over a treatment period of 6 days and the weight of tumors was determined at day 6 .
As presented in Fig. 4, the ADM $(\mathrm{P}<0.001)$, ADM-LMCC nanoparticles $(\mathrm{P}<0.001)$, and ADM-AMCC $(\mathrm{P}<0.001)$ significantly reduced the tumor volume compared with the saline group, in particular at day 6. Notably, the tumor volume of the ADM-LMCC group was the smallest $\left(679.1 \pm 128.2 \mathrm{~mm}^{3}\right)$. The histopathological changes of the tumor tissues are presented in Fig. 4. ADM-LMCC nanoparticles greatly improved the damage to tumor tissues in H22 xenograft mice; as compared with mice treated with ADM alone.

Additionally, the antitumor efficacy of ADM-loaded nanoparticles was evaluated by calculating the tumor inhibition rate of the mice following tumor implantation, compared with the saline group or ADM group. As presented in Table III, the ADM-LMCC group had the highest tumor inhibition rate at $62.7 \%$, followed by the ADM and ADM-AMCC groups at 51.2 and $42.5 \%$, respectively.

Biodistribution. The tissue distribution profiles of ADMloaded nanoparticles and ADM following intravenous administration were compared in the mice. The concentration of ADM in each tissue was determined using the fluorescence method. As presented in Fig. 5, ADM was widely and rapidly distributed into the majority of tissues following intravenous administration of the nanoparticles, and the highest 
Table III. Antitumor efficacy of ADM or ADM-loaded nanoparticle in H22 xenograft mice.

\begin{tabular}{|c|c|c|c|c|c|}
\hline \multirow[b]{2}{*}{ Drug } & \multirow[b]{2}{*}{$\begin{array}{c}\text { Dose } \\
(\mathrm{mg} / \mathrm{kg})\end{array}$} & \multicolumn{2}{|c|}{ Body weight (g) } & \multirow[b]{2}{*}{$\begin{array}{c}\text { Tumor } \\
\text { weight (g) }\end{array}$} & \multirow[b]{2}{*}{$\begin{array}{c}\text { Inhibition } \\
(\%)\end{array}$} \\
\hline & & $\begin{array}{c}\text { Prior to } \\
\text { administration }\end{array}$ & $\begin{array}{c}\text { Following } \\
\text { administration }\end{array}$ & & \\
\hline Saline & - & $24.3 \pm 1.9$ & $29.1 \pm 2.3$ & $1.29 \pm 0.34$ & - \\
\hline $\mathrm{ADM} \cdot \mathrm{HCl}$ & 5 & $24.0 \pm 0.9$ & $27.4 \pm 1.7$ & $0.63 \pm 0.10^{\mathrm{a}}$ & $51.2 \pm 7.7$ \\
\hline LMCC & 5 & $23.9 \pm 1.3$ & $26.1 \pm 2.1$ & $0.48 \pm 0.07^{\mathrm{b}}$ & $62.7 \pm 5.4$ \\
\hline AMCC & 5 & $23.3 \pm 1.2$ & $26.4 \pm 2.2$ & $0.74 \pm 0.19^{b}$ & $42.5 \pm 14.7$ \\
\hline
\end{tabular}

${ }^{\mathrm{a}} \mathrm{P}<0.01 ;{ }^{\mathrm{b}} \mathrm{P}<0.001$. ADM $\cdot \mathrm{HCl}$, Adriamycin hydrochloric acid; LMCC, lactose myristoyl carboxymethyl chitosan; AMCC, algal polysaccharide myristoyl carboxymethyl chitosan.

A

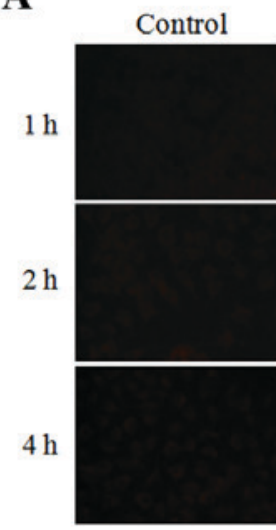

HT22

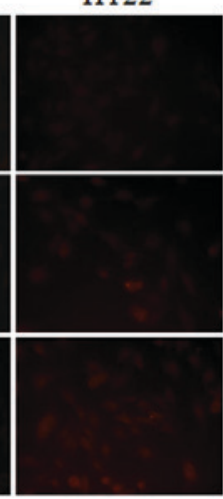

HU7

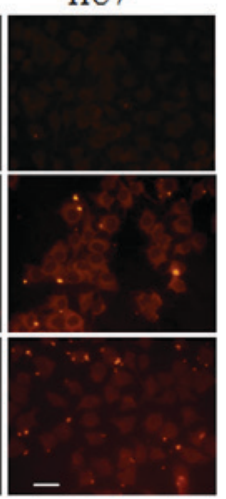

B

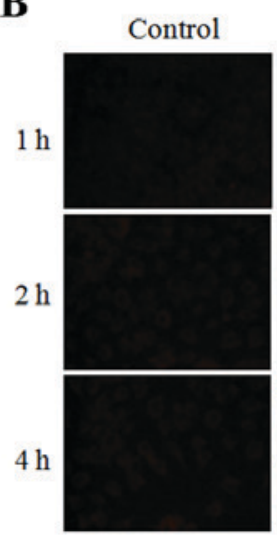

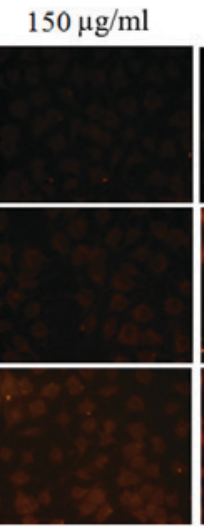
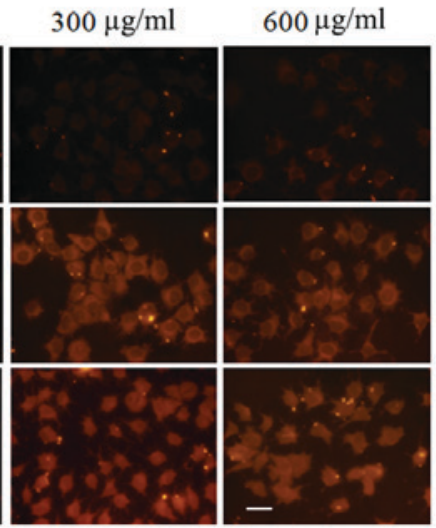

Figure 3. Fluorescence visualization of ADM intracellular accumulation. (A) HU7 and HT22 cells were treated with free RBITC solution or RBITC-LMCC nanoparticles. Fluorescent microimages of the cells were obtained at 1, 2 and $4 \mathrm{~h}$. (B) HU7 cells were treated with various concentrations (150, 300 and $600 \mu \mathrm{g} / \mathrm{ml}$ ) of RBITC-LMCC nanoparticles for 1, 2, and $4 \mathrm{~h}$, respectively. Scale bars, $10 \mu \mathrm{m}$. ADM, Adriamycin; RBITC, rhodamine B isothiocyanate.

concentration of ADM was identified in liver tissue, followed by lung, kidney, spleen, heart, brain and plasma tissues in decreasing order at $1 \mathrm{~h}$ subsequent to administration. Following $8 \mathrm{~h}$, the drug concentration was decreased in the following order: Liver $>$ lung $>$ kidney $>$ spleen $>$ brain $>$ plasma $>$ heart; ADM concentrations in the heart, brain and plasma tissues were low. However, following administration of ADM hydrochloride at $1 \mathrm{~h}$, the maximum concentration of the drug was observed in the liver, followed by the kidney and lung. The exposure of the organs to ADM at $8 \mathrm{~h}$ decreased in the following order: Kidney $>$ liver $>$ spleen $>$ lung $>$ heart $>$ plasma $>$ brain.

\section{Safety of nanoparticles and ADM-loaded nanoparticles}

Hemolysis test. The amphiphilic compounds may solubilize lipids or be inserted into phospholipid membranes to destabilize them, which may lead to hemolysis of RBC. Thus, the hemolysis test is required for amphiphilic LMCC and AMCC nanoparticles (30). The HR of LMCC and AMCC was revealed to be $<5 \%$, including at concentrations $\leq 10 \mathrm{mg} / \mathrm{ml}$, which indicates that the hemolysis of LMCC and AMCC was negligible (Table IV).

Acute toxicity. The acute toxicity evaluation in vivo was performed on the mice following the administration of nanoparticles. Following an injection of 2,000 mg/kg nanoparticles,
Table IV. Hemolysis rate of LMCC and AMCC.

\begin{tabular}{lcc}
\hline & \multicolumn{2}{c}{ Hemolysis rate (\%) } \\
\cline { 2 - 3 }$(\mathrm{mg} / \mathrm{ml})$ & LMCC & AMCC \\
\hline 2 & 0.52 & 0.74 \\
4 & 1.37 & 1.63 \\
6 & 1.79 & 2.03 \\
8 & 2.95 & 2.13 \\
10 & 4.51 & 3.97 \\
\hline
\end{tabular}

LMCC, lactose myristoyl carboxymethyl chitosan; AMCC, algal polysaccharide myristoyl carboxymethyl chitosan.

no mortalities occurred in the treatment group; however, the decrease in general distress in test mice was observed, such as inactivity, shortness of breath, and non-eating, which returned to normal $2 \mathrm{~h}$ subsequent to administration. Mortality was not observed until the end of the experiment. The autopsy of the treated mice did not reveal any macroscopic changes in the major organs, including the heart, liver, spleen, lung and kidney. 
A

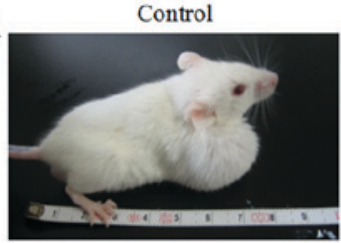

ADM-LMCC

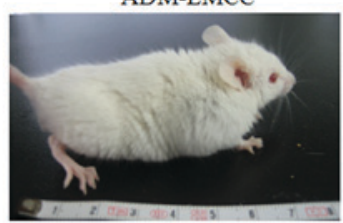

C

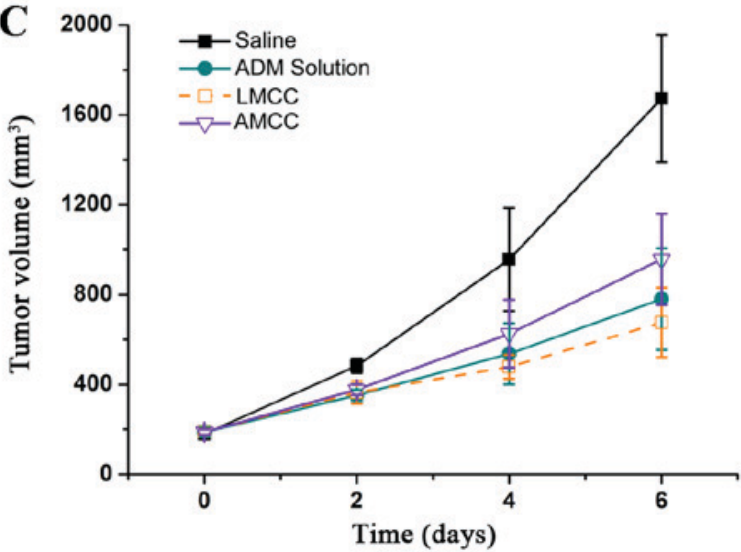

B

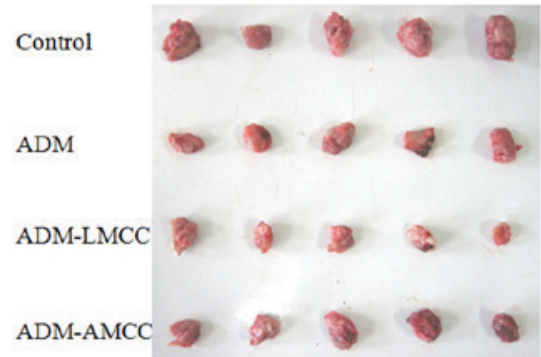

D

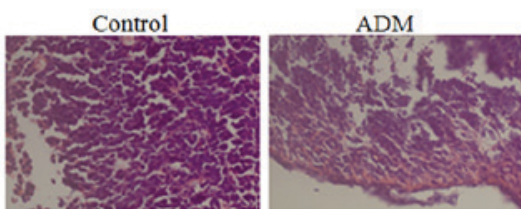

ADM-LMCC

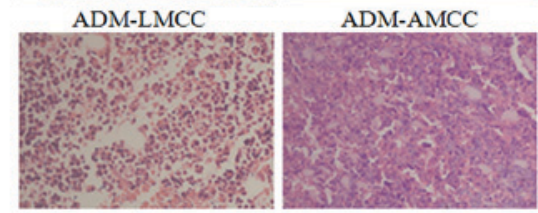

Figure 4. Antitumor activity of ADM-loaded nanoparticles in vitro. (A) Mice were inoculated subcutaneously with $\mathrm{H} 22$ cells (1x $10^{6} / \mathrm{mouse} / 0.2 \mathrm{ml}$ ). (B) Xenograft tumors were harvested and presented. (C) Tumor growth curves of various treatment groups were based on the tumor volume. Data are expressed as the mean \pm standard deviation ( $n=5$ ). (D) Histopathology of the tumor tissues of the tumor-bearing mice (x40). ADM, Adriamycin; LMCC, lactose myristoyl carboxymethyl chitosan; AMCC, algal polysaccharide myristoyl carboxymethyl chitosan.
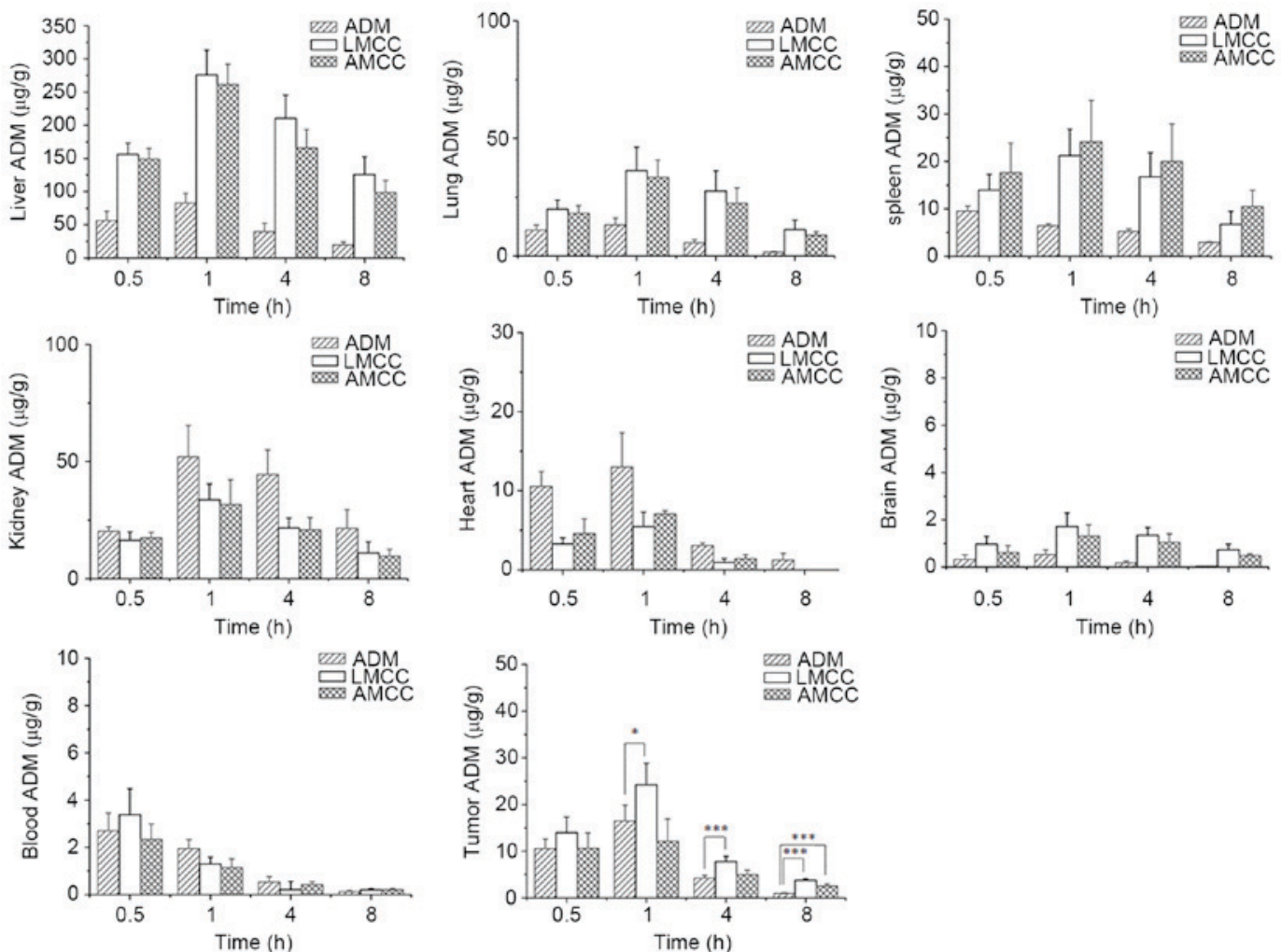

Figure 5. Tissue distribution and plasma concentration of ADM among various organs (liver, lung, spleen, kidney, heart, brain, tumor and plasma) at various sampling times following intravenous administration at a dose of $10 \mathrm{mg} / \mathrm{kg}$ in mice. Each column represents the mean \pm standard deviation $(\mathrm{n}=12)$. ${ }^{*} \mathrm{P}<0.05$, ${ }^{* *} \mathrm{P}<0.01,{ }^{* * *} \mathrm{P}<0.001$. ADM, Adriamycin; LMCC, lactose myristoyl carboxymethyl chitosan; AMCC, algal polysaccharide myristoyl carboxymethyl chitosan. 


\section{Discussion}

The present study represents the first report on the LMCC or AMCC nanoparticles as a targeted delivery system for ADM to treat HCC. The drug-loading capacity of LMCC nanoparticles was slightly higher compared with that of AMCC. This may be associated with the alternate morphologies of LMCC and AMCC. The TEM images indicated that the surface of LMCC was rough, whereas that of AMCC was uniform. The rough surface allowed for easier drug loading, due to the presence of more binding sites.

The ADM release rate from LMCC and AMCC nanoparticles was higher at $\mathrm{pH} 5.5$ compared with $\mathrm{pH} 7.4$, which indicated that the drug release behaviors of the nanoparticles were influenced by $\mathrm{pH}$. This was consistent with the ADM-loaded poly (OEGylated L-glutamate)-block-poly (L-glutamic acid) and (poly (galactosylated L-glutamate)-block-poly (L-glutamic acid) micelles with $\mathrm{pH}$-dependent release behavior (31). The tumor extracellular microenvironment is often acidic (32), thus, the $\mathrm{pH}$-dependent release behavior may contribute to tumor targeting. In addition, the TEM images indicated that the mean particle size of LMCC and AMCC were 30-50 nm. The zeta potential of these two nanoparticles was approximately -17.5 and $-18.7 \mathrm{mV}$, respectively. The particle size, negative surface charge and $\mathrm{pH}$-dependent release behavior contributed to the liver targeting of ADM.

The cellular uptake of ADM-loaded LMCC nanoparticles in HU7 cells was higher compared with HT22 cells in vitro, which indicated that ADM-loaded nanoparticles may effectively target HCC cells. In vivo, ADM-loaded LMCC was the most effective combination at suppressing tumor growth, compared with free ADM and ADM-AMCC nanomedicine in the H22 xenograft mouse model. These results suggest that the lactose modified LMCC nanoparticles may significantly improve the antitumor effect and liver targeting of ADM. Although ADM was widely distributed into the majority of tissues following intravenous administration of the nanoparticles, the highest ADM levels were revealed to be in the liver, lung, kidney and spleen. The localization of ADM to the liver was consistent with uptake by the HU7 cells, which further demonstrated that LMCC nanoparticles facilitated the effective liver targeting of ADM.

With respect to safety evaluation, nanoparticle hemolysis was negligible due to the hemolysis rate, which was $<5 \%$ at $10 \mathrm{mg} / \mathrm{ml}$. Additionally, there was no significant body weight loss, mouse mortality or organ damage following the administration of nanoparticles, compared with the initial body weights of the tumor-bearing mice during the entire experimental period. The safety evaluation results indicated that nanoparticles were well tolerated at the dosage level investigated.

In conclusion, amphiphilic LMCC and AMCC nanoparticles were developed and characterized as a liver targeting delivery system for ADM. In vitro and in vivo studies demonstrated that LMCC nanoparticles have superior antitumor capability, and higher affinity for HU7 cells and liver tissues. Furthermore, no acute toxicity or hemolysis was revealed. The results indicate that LMCC nanoparticles may be useful in the development of a liver targeting drug delivery system.

\section{Acknowledgements}

The present study was supported by the Science and Technology Project of Xuzhou City (grant no. XZZD1351), National Natural Science Foundation of China (grant no. 81102381).

\section{References}

1. Kimhofer T, Fye H, Taylor-Robinson S, Thursz M and Holmes E: Proteomic and metabonomic biomarkers for hepatocellular carcinoma: A comprehensive review. Br J Cancer 112: 1141-1156, 2015.

2. Clark T, Maximin S, Meier J, Pokharel S and Bhargava P: Hepatocellular Carcinoma: Review of epidemiology, screening, imaging diagnosis, response assessment, and treatment. Curr Probl Diagn Radiol 44: 479-486, 2015.

3. Goh GB, Chang PE and Tan CK: Changing epidemiology of hepatocellular carcinoma in Asia. Best Pract Res Clin Gastroenterol 29: 919-928, 2015.

4. Ikeda K: Current therapy for hepatocellular carcinoma. Nihon Rinsho 68: 1129-1136, 2010 (In Japanese).

5. Chu KK and Cheung TT: Update in management of hepatocellular carcinoma in Eastern population. World J Hepatol 7: 1562-1571, 2015.

6. Yang Y, Wang C, Lu Y, Bai W, An L, Qu J, Gao X, Chen Y, Zhou L, Wu Y, et al: Outcomes of ultrasound-guided percutaneous argon-helium cryoablation of hepatocellular carcinoma. J Hepatobiliary Pancreat Sci 19: 674-684, 2012.

7. Furuta M, Kozaki K, Tanimoto K, Tanaka S, Arii S, Shimamura T, Niida A, Miyano S and Inazawa J: The tumorsuppressive miR-497-195 cluster targets multiple cell-cycle regulators in hepatocellular carcinoma. PLoS One 8: e60155, 2013.

8. Mizuguchi Y, Takizawa T, Yoshida H and Uchida E: Dysregulated miRNA in progression of hepatocellular carcinoma: A systematic review. Hepatol Res 46: 391-406, 2016.

9. Guaragna A, Chiaviello A, Paolella C, D'Alonzo D, Palumbo G and Palumbo G: Synthesis and evaluation of folate-based chlorambucil delivery systems for tumor-targeted chemotherapy. Bioconjug Chem 23: 84-96, 2012.

10. Vinh NQ, Naka S, Cabral H, Murayama H, Kaida S, Kataoka K, Morikawa S and Tani T: MRI-detectable polymeric micelles incorporating platinum anticancer drugs enhance survival in an advanced hepatocellular carcinoma model. Int J Nanomedicine 10: 4137-4147, 2015.

11. Pérez-Herrero E and Fernández-Medarde A: Advanced targeted therapies in cancer: Drug nanocarriers, the future of chemotherapy. Eur J Pharm Biopharm 93: 52-79, 2015.

12. Puntawee S, Theerasilp M, Reabroi S, Saeeng R, Piyachaturawat P, Chairoungdua A and Nasongkla N: Solubility enhancement and in vitro evaluation of PEG-b-PLA micelles as nanocarrier of semi-synthetic andrographolide analogue for cholangiocarcinoma chemotherapy. Pharm Dev Technol 21: 437-444, 2016.

13. Prabaharan M: Chitosan-based nanoparticles for tumor-targeted drug delivery. Int J Biol Macromol 72: 1313-1322, 2015.

14. Wang JJ, Zeng ZW, Xiao RZ, Xie T, Zhou GL, Zhan XR and Wang SL: Recent advances of chitosan nanoparticles as drug carriers. Int J Nanomedicine 6: 765-774, 2011.

15. Tiyaboonchai W: Chitosan nanoparticles: A promising system for drug delivery. Naresuan Uni J: Sci Technol 11: 51-66, 2003.

16. Tian Q, Wang XH, Wang W, Zhang CN, Wang P and Yuan Z: Self-assembly and liver targeting of sulfated chitosan nanoparticles functionalized with glycyrrhetinic acid. Nanomedicine 8: 870-879, 2012.

17. Guan M, Zhou Y, Zhu QL, Liu Y, Bei YY, Zhang XN and Zhang Q: N-trimethyl chitosan nanoparticle-encapsulated lactosyl-norcantharidin for liver cancer therapy with high targeting efficacy. Nanomedicine 8: 1172-1181, 2012.

18. Richardson KE, Xue Z, Huang Y, Seo Y and Lapitsky Y: Physicochemical and antibacterial properties of surfactant mixtures with quaternized chitosan microgels. Carbohydr Polym 93: 709-717, 2013.

19. Huo M, Zhang Y, Zhou J, Zou A, Yu D, Wu Y, Li J and Li H: Synthesis and characterization of low-toxic amphiphilic chitosan derivatives and their application as micelle carrier for antitumor drug. Int J Pharm 394: 162-173, 2010. 
20. Chen SC, Wu YC, Mi FL, Lin YH, Yu LC and Sung HW: A novel $\mathrm{pH}$-sensitive hydrogel composed of N,O-carboxymethyl chitosan and alginate cross-linked by genipin for protein drug delivery. J Control Release 96: 285-300, 2004.

21. Ma P, Liu S, Huang Y, Chen X, Zhang L and Jing X: Lactose mediated liver-targeting effect observed by ex vivo imaging technology. Biomaterials 31: 2646-2654, 2010.

22. Sellimi S, Younes I, Ayed HB, Maalej H, Montero V, Rinaudo M, Dahia M, Mechichi T, Hajji M and Nasri M: Structural, physicochemical and antioxidant properties of sodium alginate isolated from a Tunisian brown seaweed. Int J Biol Macromol 72: $1358-1367,2015$

23. Otter M, Kuiper J, Bos R, Rijken D and van Berkel TJ: Characterization of the interaction both in vitro and in vivo of tissue-type plasminogen activator (t-PA) with rat liver cells. Biochem J 284: 545-550, 1992.

24. Puxbaum V, Nimmerfall E, Bäuerl C, Taub N, Blaas PM, Wieser J, Mikula M, Mikulits W,Ng KM, Yeoh GC and Mach L: M6P/IGF2R modulates the invasiveness of liver cells via its capacity to bind mannose 6-phosphate residues. J Hepatol 57: 337-343, 2012.

25. Bertin PA, Smith D and Nguyen ST: High-density doxorubicin-conjugated polymeric nanoparticles via ring-opening metathesis polymerization. Chem Commun (Camb) 14: 3793-3795, 2005.

26. Fontana MC, Beckenkamp A, Buffon A and Beck RC: Controlled release of raloxifene by nanoencapsulation: Effect on in vitro antiproliferative activity of human breast cancer cells. Int J Nanomedicine 9: 2979-2991, 2014
27. Yao YC, Zhan XY, Zhang J, Zou XH, Wang ZH, Xiong YC, Chen J and Chen GQ: A specific drug targeting system based on polyhydroxyalkanoate granule binding protein $\mathrm{PhaP}$ fused with targeted cell ligands. Biomaterials 29: 4823-4830, 2008.

28. Peng W, Hu C, Shu Z, Han T, Qin L and Zheng C: Antitumor activity of tatariside $\mathrm{F}$ isolated from roots of Fagopyrum tataricum (L.) Gaertn against H22 hepatocellular carcinoma via up-regulation of p53. Phytomedicine 22: 730-736, 2015.

29. Van Lancker MA, Bellemans LA and De Leenheer AP: Quantitative determination of low concentrations of adriamycin in plasma and cell cultures, using a volatile extraction buffer. J Chromatogr 374: 415-420, 1986.

30. Du P, Viswanathan UM, Xu Z, Ebrahimnejad H, Hanf B, Burkholz T, Schneider M, Bernhardt I, Kirsch G and Jacob C: Synthesis of amphiphilic seleninic acid derivatives with considerable activity against cellular membranes and certain pathogenic microbes. J Hazard Mater 269: 74-82, 2014.

31. Ding J, Xiao C, Li Y, Cheng Y, Wang N, He C, Zhuang X, Zhu X and Chen X: Efficacious hepatoma-targeted nanomedicine self-assembled from galactopeptide and doxorubicin driven by two-stage physical interactions. J Control Release 169: 193-203, 2013.

32. Kato Y, Ozawa S, Miyamoto C, Maehata Y, Suzuki A, Maeda T and Baba Y: Acidic extracellular microenvironment and cancer. Cancer Cell Int 13: 89, 2013. 